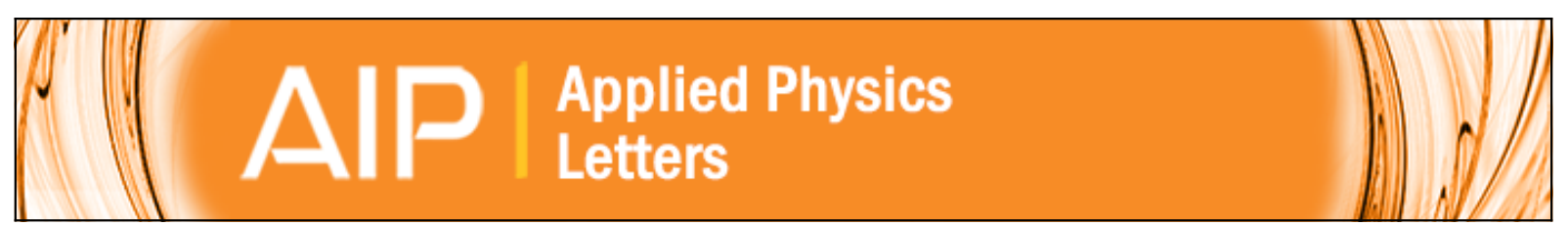

Characteristics of AIN/GaN nanowire Bragg mirror grown on (001) silicon by molecular beam epitaxy

Junseok Heo, Zifan Zhou, Wei Guo, Boon S. Ooi, and Pallab Bhattacharya

Citation: Applied Physics Letters 103, 181102 (2013); doi: 10.1063/1.4827338

View online: http://dx.doi.org/10.1063/1.4827338

View Table of Contents: http://scitation.aip.org/content/aip/journal/apl/103/18?ver=pdfcov

Published by the AIP Publishing

$\underset{\substack{\text { Publoning } \\ \text { PIP }}}{A}$ Re-register for Table of Content Alerts

Create a profile.

Sign up today! 


\title{
Characteristics of AIN/GaN nanowire Bragg mirror grown on (001) silicon by molecular beam epitaxy
}

\author{
Junseok Heo, ${ }^{1, a)}$ Zifan Zhou, ${ }^{2}$ Wei Guo, ${ }^{3}$ Boon S. Ooi, ${ }^{4}$ and Pallab Bhattacharya ${ }^{1}$ \\ ${ }^{1}$ Center for Photonics and Multiscale Nanomaterials, Department of Electrical Engineering \\ and Computer Science, University of Michigan, Ann Arbor, Michigan 48109-2122, USA \\ ${ }^{2}$ Department of Electrical and Computer Engineering, University of Michigan-Dearborn, Dearborn, \\ Michigan 48128, USA \\ ${ }^{3}$ Microsystems Engineering, Rochester Institute of Technology, Rochester, New York 14623, USA \\ ${ }^{4}$ Photonics Laboratory, King Abdullah University of Science and Technology, Thuwal 23955-6900, \\ Saudi Arabia
}

(Received 23 July 2013; accepted 15 October 2013; published online 28 October 2013)

\begin{abstract}
$\mathrm{GaN}$ nanowires containing AlN/GaN distributed Bragg reflector (DBR) heterostructures have been grown on (001) silicon substrate by molecular beam epitaxy. A peak reflectance of $70 \%$ with normal incidence at $560 \mathrm{~nm}$ is derived from angle resolved reflectance measurements on the as-grown nanowire DBR array. The measured peak reflectance wavelength is significantly blue-shifted from the ideal calculated value. The discrepancy is explained by investigating the reflectance of the nanoscale DBRs with a finite difference time domain technique. Ensemble nanowire microcavities with $\mathrm{In}_{0.3} \mathrm{Ga}_{0.7} \mathrm{~N}$ nanowires clad by $\mathrm{AlN} / \mathrm{GaN}$ DBRs have also been characterized. Room temperature emission from the microcavity exhibits considerable linewidth narrowing compared to that measured for unclad $\mathrm{In}_{0.3} \mathrm{Ga}_{0.7} \mathrm{~N}$ nanowires. The resonant emission is characterized by a peak wavelength and linewidth of $575 \mathrm{~nm}$ and $39 \mathrm{~nm}$, respectively. (C) 2013 AIP Publishing LLC. [http://dx.doi.org/10.1063/1.4827338]
\end{abstract}

The development of reliable and efficient visible light emitting diodes (LEDs) and lasers with wide bandgap semiconductors is important for solid state lighting, full color mobile projectors, displays, and medical applications. ${ }^{1-8}$ GaN-based materials and heterostructures are well-suited for such applications. However, serious materials limitations have impeded the expected progress. ${ }^{7}$ Lack of low-cost and highquality GaN substrates has necessitated epitaxy on mismatched substrates, leading to large defect densities. ${ }^{9}$ The large lattice mismatch between $\mathrm{GaN}$ and $\mathrm{AIN}$ has prevented the realization of high quality Bragg mirrors ${ }^{10}$ and low threshold vertical cavity surface emitting lasers (VCSELs) ${ }^{6}$ and electrically pumped polariton lasers, ${ }^{11,12}$ where such mirrors are needed.

$\mathrm{Ga}(\mathrm{In}) \mathrm{N}$ nanowires can be epitaxially grown without a catalyst on (001) or (111) silicon substrate in the wurtzite crystalline form with the c-axis parallel to the growth direction. ${ }^{13-15}$ They are generally free of extended defects, and the radial relaxation of strain during epitaxy results in very small piezoelectric polarization in nanowire heterostructures. ${ }^{16-18}$ The surface recombination velocity is also quite small $\left(\sim 10^{4} \mathrm{~cm} / \mathrm{s}\right){ }^{18}$ These nanowires, either as the as-grown array on silicon or as selectively dispersed single nanowires or nanowire $\mathrm{p}-\mathrm{n}$ junctions, have been used to realize visible LEDs up to $\lambda \sim 630 \mathrm{~nm},{ }^{19}$ visible electrically pumped single photon sources, ${ }^{20}$ single nanowire lasers, ${ }^{21,22}$ and room temperature optically pumped low threshold polariton lasers. ${ }^{23}$ In this work we have grown AlN/GaN nanowire distributed Bragg reflector (DBR) heterostructures ${ }^{16}$ on (001) silicon substrates. Nanowire microcavities consisting of $\mathrm{In}_{0.3} \mathrm{Ga}_{0.7} \mathrm{~N}$ nanowires clad by the DBRs were also grown

\footnotetext{
a) Present address: Department of Electrical and Computer Engineering, Ajou University, Suwon 443-749, South Korea. Electronic mail: jsheo@ajou.ac.kr.
}

and characterized. Results from angle resolved reflectance measurements made on the DBR heterostructures and photoluminescence measurements made on the microcavities are presented and discussed.

GaN nanowires with an aerial density of $\sim 10^{11} \mathrm{~cm}^{-2}$ were grown on (001) Si substrate in a molecular beam epitaxy (MBE) system. After removal of the surface oxide on the substrate with a $900^{\circ} \mathrm{C}$ anneal in the growth chamber, the temperature is lowered to $800^{\circ} \mathrm{C}$, and a few monolayers of $\mathrm{Ga}$ are deposited with a Ga flux of $1.3 \times 10^{-7}$ Torr in the absence of $\mathrm{N}$. GaN nanowire growth is initiated at the same temperature at a rate of $300 \mathrm{~nm} / \mathrm{h}$ under N-rich conditions. The Ga flux is maintained at $1.3 \times 10^{-7}$ Torr, and the nitrogen flow rate is held constant at $1 \mathrm{sccm}$. In order to grow only the AlN/GaN DBR heterostructure, a $200 \mathrm{~nm}$ seed GaN nanowire is first grown under the conditions outlined above and 22 pairs of AlN/GaN quarter-wave layers are grown at $800^{\circ} \mathrm{C}$ with $\mathrm{Al}$ and Ga fluxes of $1.5 \times 10^{-7}$ Torr and $1.3 \times 10^{-7}$ Torr, respectively. To realize a nanowire microcavity, $250 \mathrm{~nm} \operatorname{In}_{0.3} \mathrm{Ga}_{0.7} \mathrm{~N}$ nanowires are grown and are clad by 22 and 3 pairs of bottom and top AlN/GaN DBR, respectively. A detailed description of $\mathrm{InGaN}$ nanowire growth is provided in our previous publication. $^{15}$

The structural properties of the nanowires were investigated by scanning electron microscope (SEM) and highresolution transmission electron microscope (HR-TEM) imaging. As shown in Fig. 1(a)), a high density of GaN nanowires can be grown on (001) Si substrate with an average diameter of $\sim 40 \mathrm{~nm}$ and with excellent uniformity in length. The TEM image of Fig. 1(b) shows a GaN nanowire with AlN/GaN DBR heterostructures in which multiple AlN/GaN Bragg pairs $(77 / 71 \mathrm{~nm})$ were grown along the c-axis. The image depicts sharp AlN/GaN interfaces with an absence of extended defects. The insets of Fig. 1(b) show the selected 


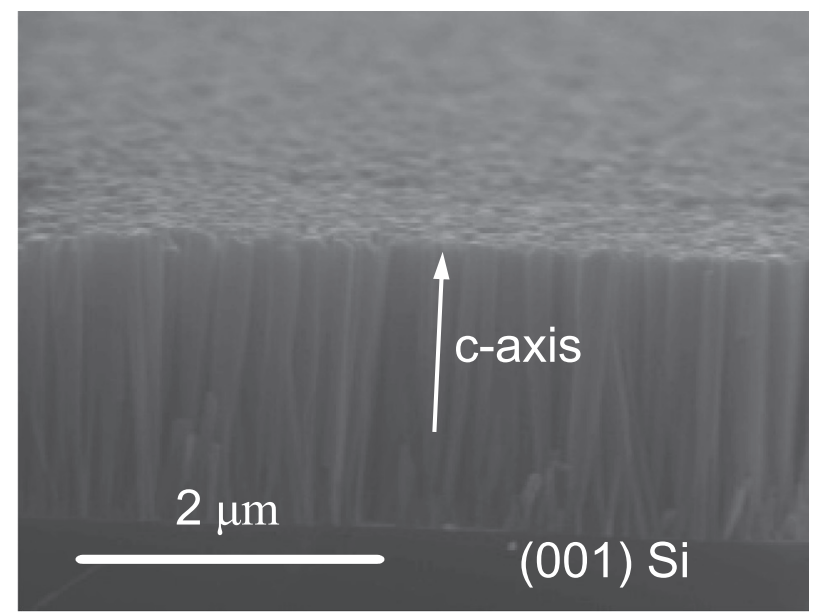

(a)

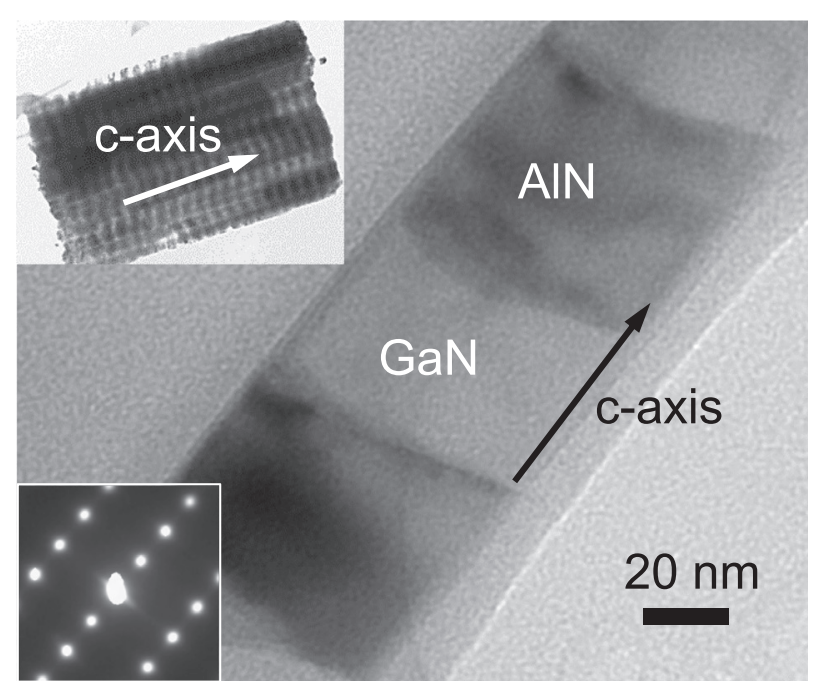

(b)

FIG. 1. (a) High density $\left(\sim 10^{11} \mathrm{~cm}^{-2}\right) \mathrm{GaN}$ nanowires grown by molecular beam epitaxy on (001) Si; (b) TEM image of GaN nanowire with AlN/GaN DBR depicting the smooth interface between the AlN and GaN layers. The insets show the selected area diffraction pattern of the $\mathrm{GaN}$ nanowire region and an array of nanowires with DBR heterostructures, respectively.

area diffraction (SAD) pattern of the GaN region and a TEM image of an ensemble of nanowires with AIN/GaN DBR heterostructures at low magnification, respectively. The SAD pattern reveals that the $c$-plane of the nanowire is normal to the growth direction.

The optical properties of the as-grown nanowire DBR structures in which the thickness of AlN and GaN are 77 and $71 \mathrm{~nm}$, respectively, were investigated by angle resolved reflectance measurement with a spot size of $3 \mathrm{~mm}$. As shown in Fig. 2(a), as the incident angle increases the peak reflectance moves towards shorter wavelengths (higher energy) due to an additional in-plane wavevector normal to the growth direction. Unlike a planar DBR, each layer of the nanowire DBR structure is not homogeneous along the inplane direction due to free space between the nanowires. This results in a reduced reflectance at higher incident angles because of a non-zero in-plane wavevector of incident light.

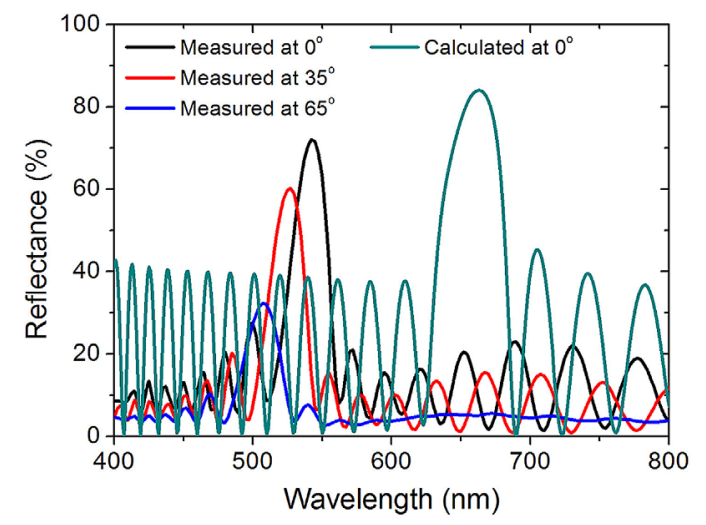

(a)

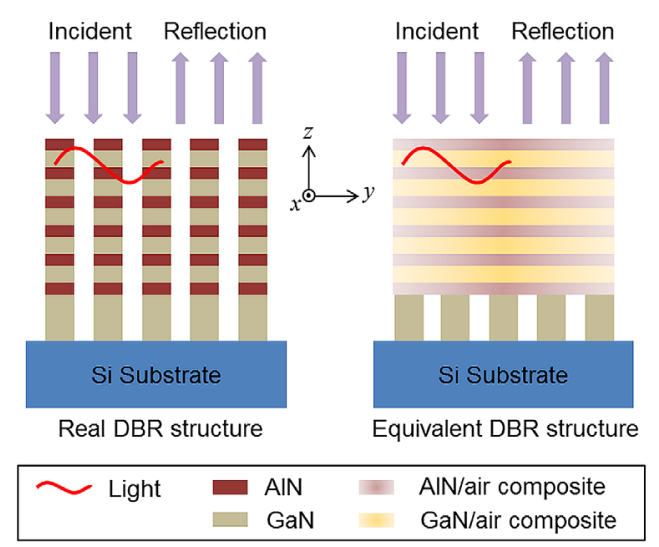

(b)

FIG. 2. (a) Measured and calculated reflectance spectra of 22 pairs of nanowire AlN/GaN DBR heterostructure at different incidence angles. The thickness of the AlN and GaN layers are 77 and $71 \mathrm{~nm}$, respectively; (b) schematic of equivalent DBR structure by considering the GaN/air and AlN/air composite materials for the constituent layers.

A maximum peak reflectance of $70 \%$ at $560 \mathrm{~nm}$ was measured at normal incidence with a stopband width of $30 \mathrm{~nm}$. For comparison, the normal incidence reflectance spectrum of a planar DBR with the same layer thickness was calculated by the transfer matrix method (TMM) ${ }^{24}$ and is also shown in the figure. It is worth noting that the measured peak wavelength of the nanowire sample exhibits a $\sim 100 \mathrm{~nm}$ blue shift from the calculated peak wavelength of a planar DBR. Moreover, the measured peak reflectance of the nanowire DBR is smaller than the calculated value for the planar DBR. Because the nanowire diameter is much smaller than the effective incident wavelength, the incident light will be weakly confined in the nanowires and will also be coupled to adjacent nanowires via the air gaps, resulting in a reduced effective refractive index of each DBR layer. In other words, as illustrated in Fig. 2(b), the incident wavefront encounters the equivalent planar GaN/air and AlN/air composite materials with smaller effective refractive index than those of $\mathrm{GaN}$ and AIN DBR layers in the nanowires. It is found from TMM analysis that the reflectance spectrum depends on the refractive index, and thickness of the constituent layers and the peak reflectance wavelength is given by $\lambda_{0}=4 d n$, where $n$ is the refractive index and $d$ is the layer thickness. ${ }^{24}$ Therefore, it is concluded that the measured blue shift of the 
peak reflectance wavelength is due to the reduced refractive index of each DBR layer.

The nanowire DBR structure has been more accurately analyzed by the 3D finite difference time domain (FDTD) method. A hexagonal close packed nanowire DBR array with a diameter of $40 \mathrm{~nm}$ and periodic boundary conditions is considered in the simulation. Since the light propagates and reflects back along the c-axis parallel to the growth direction, ordinary refractive indices of 2.41 and 2.16 were chosen for GaN and AlN, respectively. ${ }^{25}$ In order to eliminate any spurious effects due to the inadvertent formation of a photonic crystal, the positions of nanowires are slightly randomized with a maximum offset of $\sim 7 \mathrm{~nm}$ from the periodic locations, without changing the average distances between the nanowires $(\sim 45 \mathrm{~nm})$. The simulation was repeated on 10-15 different sets of randomly positioned nanowires. It is found that there is no meaningful variation among them in terms of the reflectance peak position and the stopband width. Figure 3(a) shows the electric field $\left(E_{\mathrm{y}}\right)$ distribution in the nanowires from which it is confirmed that the electromagnetic field is not well-confined in the nanowires but couples into the air gaps between the nanowires. Figure 3 (b) shows that the calculated reflectance spectrum is quantitatively in good agreement with the measured data with respect to the spectral peak position and the stopband width. The relatively smaller reflectance in the measured data is believed to be due to the misalignment of each DBR layer

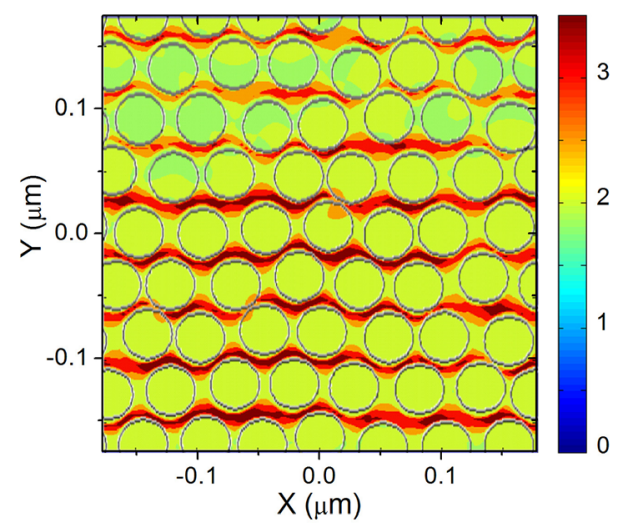

(a)

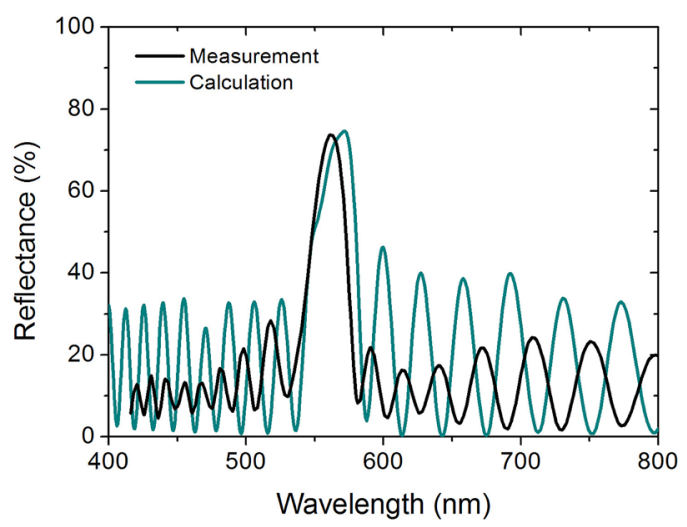

(b)

FIG. 3. (a) Calculated electrical field distribution in nanowire DBR structure calculated by FDTD method; (b) calculated and measured reflectance spectra at normal incidence for 22-pair AlN/GaN nanowire DBR. between the nanowires, as seen in the TEM image of the inset of Fig. 1(b).

Finally, nanowire microcavities with $\operatorname{In}_{0.3} \mathrm{Ga}_{0.7} \mathrm{~N}$ active region clad by 3 and 22 pairs of top and bottom DBR, respectively, were characterized by photoluminescence (PL) measurements. The microcavity was optically excited at room temperature with a continuous wave $(\mathrm{CW}) \mathrm{He}-\mathrm{Cd}$ laser at $325 \mathrm{~nm}$. The emission spectrum was analyzed with a $0.75 \mathrm{~m}$ high-resolution monochromator and detected with a photomultiplier tube using phase lock-in amplification. For comparison, control nanowires having the same indium composition, without the DBRs, were also grown and characterized. As shown in Fig. 4(a), the luminescence from the nanowires is characterized by a Gaussian lineshape with the peak at $\sim 596 \mathrm{~nm}$ and a linewidth of $\sim 78 \mathrm{~nm}$. In contrast, the output spectrum of the nanowire microcavity with DBRs exhibits several resonant peaks over the uncoupled broad background light. The most prominent peak is observed at $575 \mathrm{~nm}$, and the linewidth of this peak is estimated to be $\sim 39 \mathrm{~nm}$, after elimination of the background emission. This linewidth corresponds to a cavity quality (Q) factor of only 25 , which is due to inefficient reflection at 3 pairs of the top DBR. It is also noted that the overall emission spectrum of the nanowire microcavity with DBRs shows a small blue

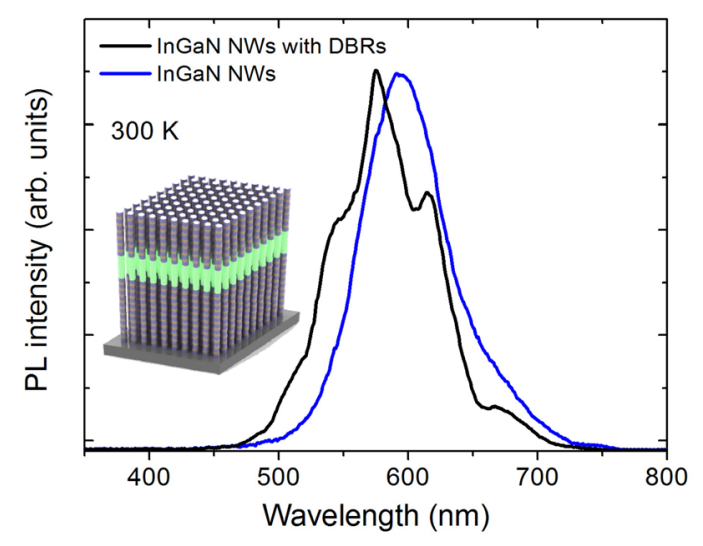

(a)
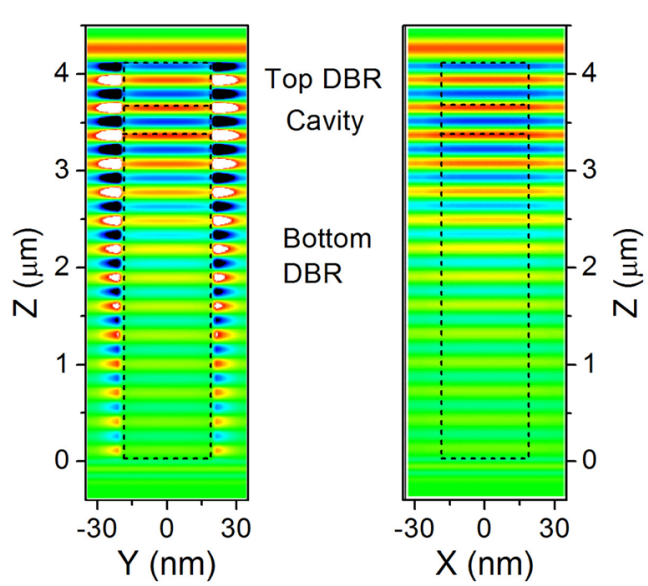

(b)

FIG. 4. (a) Room temperature photoluminescence spectra on InGaN nanowire with and without DBR cavity. Resonant peaks are observed in the PL spectrum of the microcavity; (b) calculated field profile in $y z$ - and $x z$-planes. 
shift in comparison to that of the nanowire microcavity without DBRs. This may be due to inter-diffusion of indium during the growth of the top DBR at a high temperature. The microcavity was also analyzed by 3D FDTD simulation and the calculated field $\left(E_{\mathrm{y}}\right)$ profile in $y z$ - and $x z$-planes is shown in Fig. 4(b). While the field is well confined, and the light propagation is inhibited by the bottom DBR, the field propagates freely through the top DBR, as discussed above, resulting in a small $\mathrm{Q}$ factor. Thus, with further improvement in growth of the nanowire DBR cavities and a design to enhance the cavity $Q$ factor (with more DBR periods at the top), single or ensemble green nanowire lasers on silicon substrates can be realized.

In conclusion, we have characterized AlN/GaN nanowire DBR heterostructures grown by MBE on (001) silicon substrates. High-resolution TEM images reveal sharp heterostructure interfaces with no evidence of the presence of extended defects. A peak reflectance of $70 \%$ is obtained from normal incidence reflectivity measurements on the nanowire array. Resonant cavities were grown with $\mathrm{In}_{0.3} \mathrm{Ga}_{0.7} \mathrm{~N}$ nanowires clad by 3 and 22 pairs of AlN/GaN. Resonant peaks are observed in the luminescence spectra of the cavities with a peak at $575 \mathrm{~nm}$ characterized by a linewidth of $\sim 39 \mathrm{~nm}$. With full DBRs and more optimized growth these nanostructures can become important components in nanowire-based optoelectronics.

The work was supported by a grant from KAUST, Saudi Arabia. The work was also supported by the University of Michigan-Dearborn.

${ }^{1}$ H. Ohta, S. P. DenBaars, and S. Nakamura, J. Opt. Soc. Am. B 27, B45 (2010).

${ }^{2}$ K. J. Jeon, S.-J. Kim, K. K. Park, J.-W. Kim, and G. Yoon, J. Biomed. Opt. 7, 45 (2002).

${ }^{3}$ J. M. Conway, K. H. Norris, and C. E. Bodwell, Am. J. Clin. Nutr. 40, 1123 (1984).
${ }^{4}$ C.-G. Ahn, C. S. Ah, T.-Y. Kim, C. W. Park, J.-H. Yang, A. Kim, and G. Y. Sung, Appl. Phys. Lett. 97, 103703 (2010).

${ }^{5}$ L. J. Walsh, Aust. Dent. J. 48, 146 (2003).

${ }^{6}$ S. Nakamura, Annu. Rev. Mater. Sci. 28, 125 (1998).

${ }^{7}$ S. Nakamura, T. Mukai, and M. Senoh, J. Appl. Phys. 76, 8189 (1994).

${ }^{8}$ A. Kikuchi, M. Kawai, M. Tada, and K. Kishino, Jpn. J. Appl. Phys. 43, L1524 (2004).

${ }^{9}$ I. Akasaki and H. Amano, Jpn. J. Appl. Phys. 36, 5393 (1997).

${ }^{10}$ L. E. Rodak, C. M. Miller, and D. Korakakis, Superlattice Microstruct. 49, 52 (2011)

${ }^{11}$ P. Bhattacharya, B. Xiao, A. Das, S. Bhowmick, and J. Heo, Phys. Rev. Lett. 110, 206403 (2013).

${ }^{12}$ C. Schneider, A. Rahimi-Iman, N. Y. Kim, J. Fischer, I. G. Savenko, M. Amthor, M. Lermer, A. Wolf, L. Worschech, V. D. Kulakovskii, I. A. Shelykh, M. Kamp, S. Reitzenstein, A. Forchel, Y. Yamamoto, and S. Hofling, Nature 497, 348 (2013).

${ }^{13}$ K. A. Bertness, A. Roshko, N. A. Sanford, J. M. Barker, and A. V. Davydov, J. Cryst. Growth 287, 522 (2006).

${ }^{14}$ C. Chèze, L. Geelhaar, O. Brandt, W. Weber, H. Riechert, S. Münch, R. Rothemund, S. Reitzenstein, A. Forchel, T. Kehagias, P. Komninou, G. Dimitrakopulos, and T. Karakostas, Nano Res. 3, 528 (2010).

${ }^{15}$ W. Guo, M. Zhang, A. Banerjee, and P. Bhattacharya, Nano Lett. 10, 3355 (2010).

${ }^{16}$ J. Ristić, E. Calleja, A. Trampert, S. Fernández-Garrido, C. Rivera, U. Jahn, and K. H. Ploog, Phys. Rev. Lett. 94, 146102 (2005).

${ }^{17}$ E. Calleja, J. Ristić, S. Fernández-Garrido, L. Cerutti, M. A. SánchezGarcía, J. Grandal, A. Trampert, U. Jahn, G. Sánchez, A. Griol, and B. Sánchez, Phys. Status Solidi B 244, 2816 (2007).

${ }^{18}$ J. B. Schlager, K. A. Bertness, P. T. Blanchard, L. H. Robins, A. Roshko, and N. A. Sanford, J. Appl. Phys. 103, 124309 (2008).

${ }^{19}$ S. Jahangir, M. Mandl, M. Strassburg, and P. Bhattacharya, Appl. Phys. Lett. 102, 071101 (2013).

${ }^{20}$ S. Deshpande, J. Heo, A. Das, and P. Bhattacharya, Nat. Commun. 4, 1675 (2013).

${ }^{21}$ J. Heo, W. Guo, and P. Bhattacharya, Appl. Phys. Lett. 98, 021110 (2011).

${ }^{22}$ J. B. Schlager, N. A. Sanford, K. A. Bertness, and A. Roshko, J. Appl. Phys. 109, 044312 (2011).

${ }^{23}$ J. Heo, S. Jahangir, B. Xiao, and P. Bhattacharya, Nano Lett. 13, 2376 (2013).

${ }^{24} \mathrm{M}$. Born and E. Wolf, Principles of Optics: Electromagnetic Theory of Propagation, Interference and Diffraction of Light (Cambridge University Press, 1999).

${ }^{25}$ M. Bass, C. DeCusatis, J. Enoch, V. Lakshminarayanan, G. Li, C. MacDonald, V. Mahajan, and E. V. Stryland, Handbook of Optics: Optical Properties of Materials, Nonlinear Optics, Quantum Optics, 3rd ed. (McGraw Hill Professional, 2009). 